\title{
The Implementation of A Vehicle Techniques Network Platform
}

\author{
Guoliang Dong ${ }^{1, a^{*}}$ \\ ${ }^{1}$ Research Institute of Highway Ministry of Transport, Beijing, China \\ aGL.DONG@rioh.cn
}

Keywords: Network platform, Vehicle techniques, Vehicle manufacturers, Repair workshops, Data conversion, VBA, Excel, Word

\begin{abstract}
Vehicle technique information is important for the repair workshops. A non-profit web site is built to publish the resources acquisition of each vehicle which is provided by the manufacturers. The platform composition is briefly described and a method of data conversion with VBA technique is implemented.
\end{abstract}

\section{Introduction}

Vehicle technique information is important for the repair workshops [1,2]. Network techniques have been applied in the vehicle industry. Many platform methods have been discussed $[3,4,5]$ and many web sites have been built to server the repair workshops $[6,7,8,9]$. The mistake or imperfect materials will result in repairing fault or lost. In order to get the most benefit, the motor manufacturers keep the key technique material a secret. The latest released rules require the manufacturers should disclose the technique information to the public. A web site is built to publish the resources acquisition of each vehicle which means the public can get all the repair information for the vehicle.

\section{Platform Functions}

The platform is non-profit public which is built to manage the resources acquisition for a vehicle in-used. Manufacturers should submit enterprise information, basic vehicle type information and the access to techniques information online. The site releases the access ways for each vehicle type to the public users free. The overall framework is shown in Fig. 1.

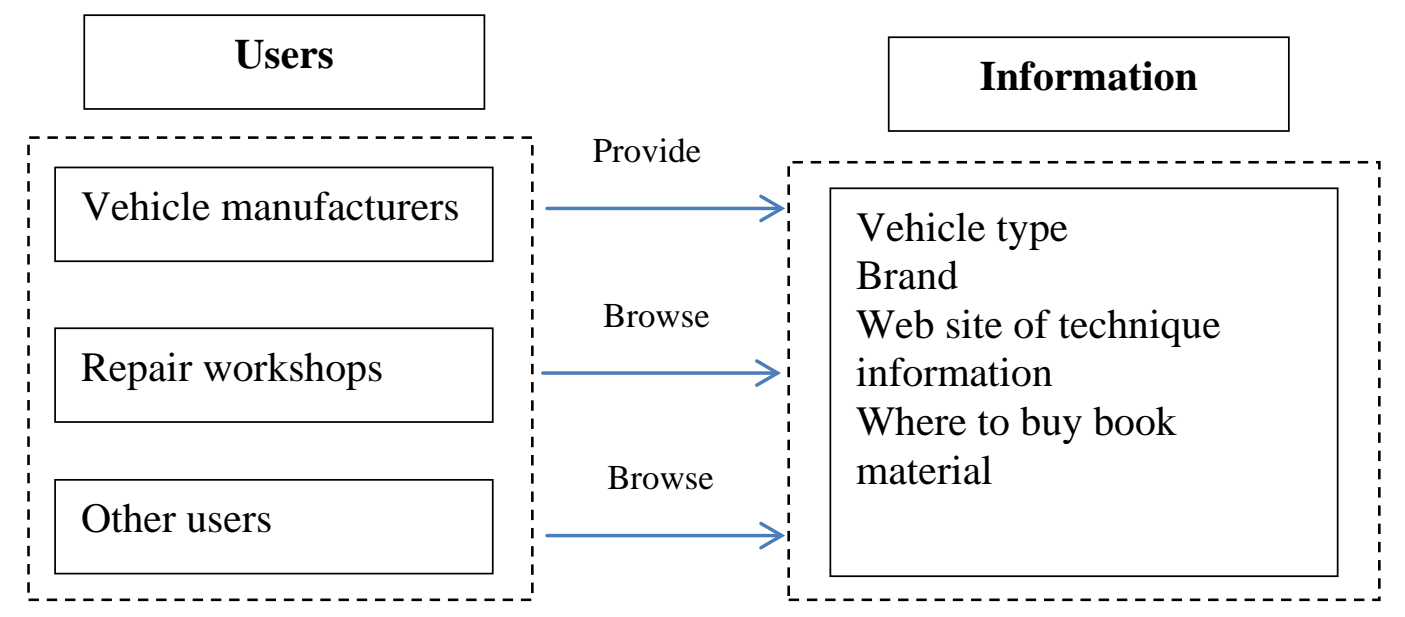

Fig.1. The users and information of the platform

Platform Users. The platform mainly serves for vehicle manufacturers and the public users such as repair workshops and vehicle owners. Vehicle manufacturers logon in the site to update basic information of vehicle types and the resources acquisition of vehicle techniques for each type. The public users freely browse and search the site to find where or how to get or buy the vehicle techniques materials from the manufacturers.

Vehicle Manufacturers. Vehicle manufacturers are the main data provider for the site. Once the platform is deployed and online the first thing they should do is to upload basic information and 
resources acquisition of the existing vehicle type. The manufacturers' qualification should be identified before they update data and the data should be in a uniform format. For the first round huge amounts of data should be imported and the left time is not much time before the public users browse the platform. In order to increase efficiency, batch importing function is developed and data are converted to a standard form by an Excel file embedded VBA codes. To decrease the failure of data updating, the first round data is provided directly to the data engineers of the web site and batch uploaded to the platform.

Basic Information Of A Vehicle Type. The basic information of a vehicle type includes enterprise name, brand, vehicle type, publicity type, acquisition ways of vehicle techniques materials, etc. The public users could search the above information by inputting a vehicle type as a keyword.

Publicity Type/Acquisition Of Vehicle Technique. Publicity type of a vehicle type can be found from the platform. Techniques information is released online or in paper provided by the manufacturers. The public users can search some of the vehicle techniques information from the manufacturer's web site.

Data Preparation For Platform Deployment. Before the site is open, plenty of data should be processed. All sorts of materials saved in Word files should be converted to a standard Excel format which will be imported into the platform database later [10]. Data engineers should process the materials. An Excel file embedded VBA codes [11] can easily handle these Word or Excel files and free data engineers from the hard and time-exhausted work of heterogeneous data transformation.

Extract Manufactures Information. The information provided by the manufactures should include the name, enterprise type, phone, address etc. The information should be checked and only the qualified enterprises can access site. Data engineers process the Word files provided by the qualified manufacturers, extract information form the tables, save them as an Excel file by a template format file and import them to the platform database. The main VBA codes embedded in an Excel [12] to implement the above function are list as below.

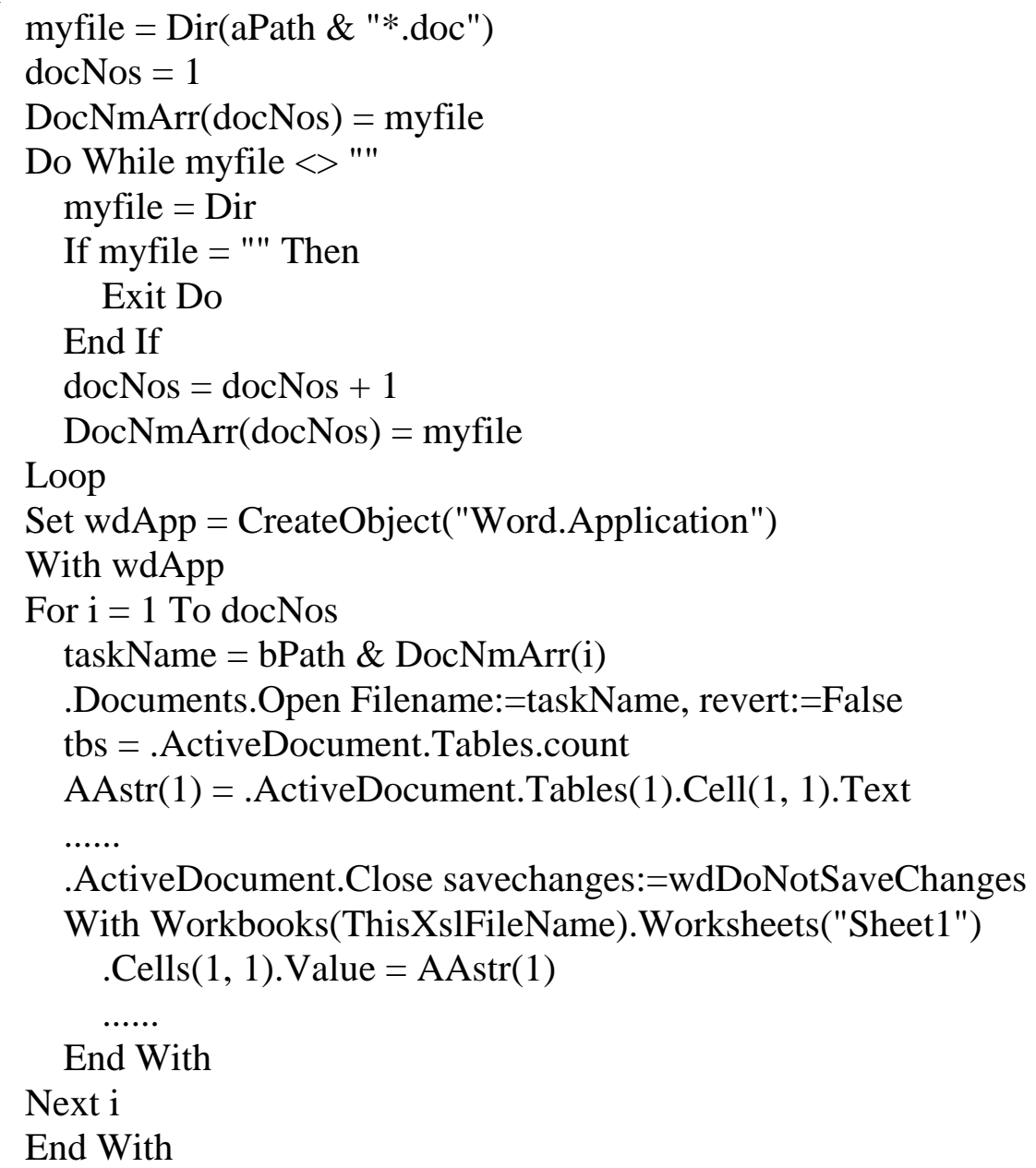


Convert Application Information. Application data are saved in the table of a Word file. Information should be extracted from the tables [13] and be converted to Excel [14] which will be imported into the platform database. The main VBA codes are list as below.

With wdApp

For $\mathrm{i}=1$ To docNos

.Documents.Open Filename:= bPath \& DocNmArr(i), revert:=False

With .ActiveDocument

Set xlapp = CreateObject("Excel.application")

xlapp. Visible $=$ True

Set appbook = xlapp. Workbooks.Add

wdApp.ActiveDocument.Select

wdApp.Selection.Tables(1).Select

wdApp.Selection.Copy

appbook.Worksheets(1).Range("A1").Paste

appbook.SaveAs bPath \& DocNmArr(i) \& ".xls", FileFormat:=xlExcel8

xlapp.DisplayAlerts $=$ True

xlapp.Quit

End With

Next i

End With

\section{Conclusions}

The vehicle techniques web site builds a bridge between the manufactures and the public users. The platform function and composition is brief described. A data transformation method for deployed database is implemented with VBA techniques. The method is proved well increasing the efficiency and decreasing the failure of data transformation.

\section{References}

[1] Sun Tao. The condition of the motor vehicle repair industry [J]. Journal of Yangtze University (Social Sciences Edition), 2012(5)79-80.

[2] Hong Yong-jie. The problems and countermeasure of the motor vehicle repair industry [J]. Journal of Taiyuan Urban Vocational College, 2015(5)157-159.

[3] Sun Ji-ming, Guo Qi-nan. Initial Analysis of Telematics Services and Platform [J]. Designing Techniques of Posts and Telecommunications, 2013(1)88-92.

[4] Xu Hai, Zhang Yu, Su Wei-dong . Development Status of Chinese Automotive Informationization Technology [J]. Auto Engineer, 2010(9)21-23.

[5] Yan Shen-jing, Nie Xiong,Wang Hao-hao, Lin Hua. Design of car information Web based on ASP technology [J]. Journal of Guangxi University (Natural Science Edition), 2005(S2)4-6.

[6] Chen Kai-hui,Li Chun.The Design and Realization of Automobile Enterprise Electronic Commerce Website [J]. Journal of Yulin Normal University, 2013 (2)143-151.

[7] Yue Liu-zhen, Lin Zu-qing . Development and Management of Vehicle Service Information based on Database . Shanghai Auto, 2014(6)29-33.

[8] Pang Zhi-nian ,Niu Di. Design of Information System Network Platform [J]. Computer \& Network, 2013(12) 62-65.

[9] Information on http://www.tjqcwx.com/. 
[10] Peng Lei,Li Xian-guo. Design and implementation of Excel mass data importing system [J]. Modern Electronics Technique, 2014(14)57-59.

[11] Gao Zhi-chao and Zhang Zhi-kui: Importing Excel into MySQL, Computer Programming Skills \& Maintenance, 2012(19) 39-40.

[12]Yunzhou Stiduo. Excel 2000 VBA [M]. Posts \& Telecom Press, 2000.

[13]Microsoft Corporation. Microsoft Word Help.

[14]Microsoft Corporation. Microsoft Excel 2000 Language Reference [M]. Beijing Hope Electronic Press, 1999. 